Copyright by the American Institute of Physics (AIP). Xing, Zengping; Li, Jiefang; Viehland, D., "Noise and scale effects on the signal-to-noise ratio in magnetoelectric laminate sensor/detection units," Appl. Phys. Lett. 91, 182902 (2007); http:// dx.doi.org/10.1063/1.2804118

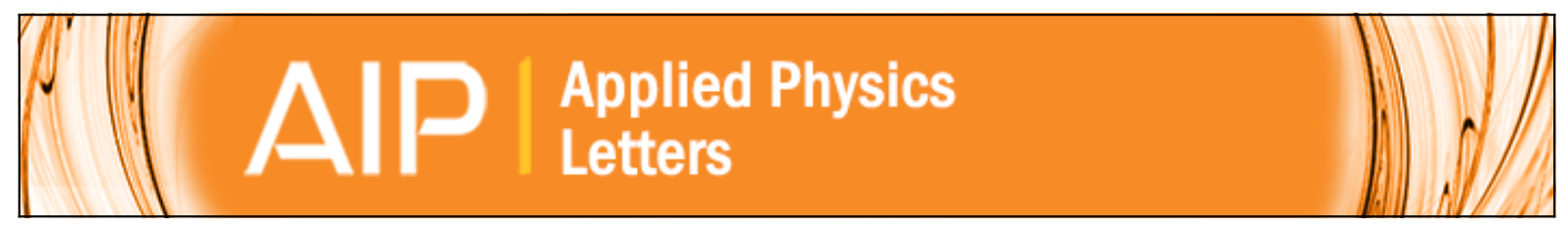

Noise and scale effects on the signal-to-noise ratio in magnetoelectric laminate sensor/detection units

Zengping Xing, Jiefang Li, and D. Viehland

Citation: Applied Physics Letters 91, 182902 (2007); doi: 10.1063/1.2804118

View online: http://dx.doi.org/10.1063/1.2804118

View Table of Contents: http://scitation.aip.org/content/aip/journal/apl/91/18?ver=pdfcov

Published by the AIP Publishing

Over 700 papers \&

presentations on

multiphysics simulation vew now

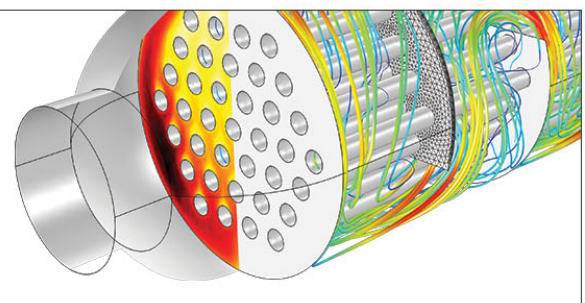




\title{
Noise and scale effects on the signal-to-noise ratio in magnetoelectric laminate sensor/detection units
}

\author{
Zengping Xing, ${ }^{\text {a) }}$ Jiefang Li, and D. Viehland \\ Materials Science and Engineering, Virginia Tech, Blacksburg, Virginia 24061, USA
}

(Received 28 August 2007; accepted 10 October 2007; published online 30 October 2007)

\begin{abstract}
We have investigated the output noise level $\left(E_{\mathrm{no}}\right)$ and signal-to-noise ratio (SNR) for various magnetoelectric (ME) laminate composites. Our findings show (i) that $E_{\text {no }}$ is nearly independent of materials couple or operational mode and practically determined by the laminate capacitance $C$ and (ii) that the SNR has a scale effect, where it is limited by the volume $(V)$ of the piezoelectric layer. Relations between SNR, $V$, and the magnetoelectric coefficient $\left(\alpha_{\mathrm{ME}}\right)$ give insights into how to construct ME sensors with enhanced sensitivity to small magnetic variation. (C) 2007 American Institute of Physics. [DOI: 10.1063/1.2804118]
\end{abstract}

The magnetoelectric (ME) effect-the induction of magnetization by an applied electric field $(E)$ or a polarization by an applied magnetic field $(H)$ (Ref. 1) - has been of recent research interests. For potential application in magnetic sensors, investigations have focused on composites of magnetostrictive layers laminated to piezoelectric ones. When an ac magnetic field $\left(H_{\mathrm{ac}}\right)$ is applied to these laminates, the magnetostrictive layer(s) elastically forces the piezoelectric layer(s) to strain, generating a piezoelectric charge. Prior studies have compared the performance of various ME laminate composites based on a materials parameter: the ME voltage coefficient $\alpha_{\mathrm{ME}}{ }^{2-9}$

Recently, a ME laminate sensor technology including an equivalent model, detection circuitry, and noise mitigation was developed. ${ }^{10,11}$ Electromagnetic shielding and grounding notably reduced environmental noise. ${ }^{11,12}$ Prototypes of passive magnetic field sensor units were developed, which were shown capable of nano-Tesla detection at quasistatic frequencies. ${ }^{11}$ Here, we will show how the signal-to-noise ratio (SNR) of ME detection units can be increased by design considerations that take into account the laminate's intrinsic noise sources.

We begin by introducing an equivalent circuit model for a ME laminate, ${ }^{10}$ as illustrated in Fig. 1, where $i_{n}$ is the spectral current noise density, $C$ is the laminate capacitance, $R$ is the resistance, $\alpha_{0} \varepsilon^{-j \phi}$ is the ME charge coefficient $\alpha_{\text {me }}$, and $H$ is the input magnetic field. We constructed a charge amplifier detection method, which introduced an equivalent noise level of $i_{C}<4 \mathrm{fA}_{\mathrm{p} \text {-p }}$. referred to the input. Since the total noise of the ME laminate that we studied was typically $\geqslant 20 \mathrm{fA}_{\text {p.-p. }}$, equivalent noise of the detection circuitry can be neglected as, at most, it will introduce a $5 \%$ error into the estimates of the total noise (noise summation rule). ${ }^{13}$ The remaining noise sources are intrinsic ones, notably (i) radiation noise $i_{G}$, (ii) dielectric loss noise $i_{D}$, and (iii) Johnson noise $i_{R}{ }^{14-17}$

Radiation noise is proportional to the square root of the thermal conductance $G$ between the ME laminate and the heat sink. Proper design of the ME detection system can significantly reduce it. Here, we used a double-stick foam bar to mount the laminate sensors to reduce the thermal conductance $G$. As a result, the radiation noise was negligible, com-

${ }^{a)}$ Electronic mail: xing@vt.edu pared to that introduced by dielectric loss and Johnson noise. The spectral current density of dielectric loss noise can be estimated as ${ }^{15,16}$

$$
i_{d}=\sqrt{4 k T \omega C \tan \delta}
$$

where $k$ is the Boltzmann constant, $T$ the absolute temperature, $\omega$ the angular frequency, $C$ the ME sensor capacitance, and $\tan \delta$ the loss tangent of the piezoelectric layer. The spectral current density of Johnson noise, which comes from the thermal agitation of electrons in a conductor, can be estimated by Nyquist's relation, ${ }^{14,15}$

$$
i_{r}=\sqrt{\frac{4 k T}{R}}=\sqrt{\frac{4 k T}{\rho \varepsilon} C},
$$

where $R$ is the resistance, $\rho$ the volume resistance, and $\varepsilon$ the dielectric constant.

Assuming that the dielectric loss and Johnson noises are uncorrelated, the total intrinsic spectral current noise density $i_{t}$ can be obtained by ${ }^{13}$

$$
i_{t}(\omega)=\sqrt{i_{d}^{2}+i_{r}^{2}}=\sqrt{4 k T C\left(\frac{1}{\rho \varepsilon}+\omega \tan \delta\right)}=A(\omega) \sqrt{C}
$$

If we assume that the noise gain of the detection circuitry is $Z_{n}(j \omega)$ and that the noise bandwidth is $f_{H^{-}} f_{L}$ (where $f_{H}$ and $f_{L}$ are the upper and lower limits of the noise band), then the total output rms voltage noise $\left(E_{\mathrm{no}}\right)$ is ${ }^{18}$

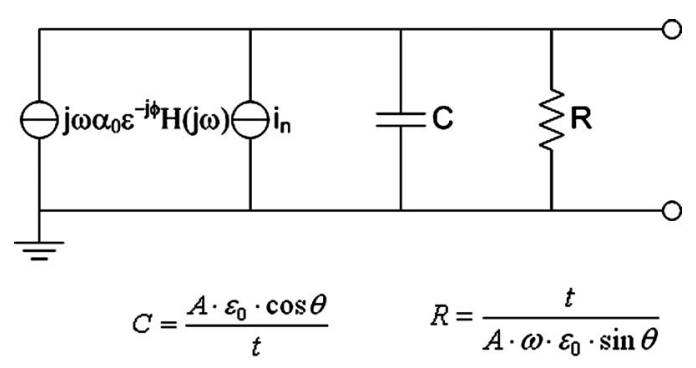

FIG. 1. Equivalent circuit model for low frequency ME laminate sensor including noise spectra considerations (Ref. 10). 
TABLE I. ME laminate sensor.

\begin{tabular}{cccccc}
\hline \hline No. & Material & Mode $^{\mathrm{a}}$ & $\begin{array}{c}C \\
(\mathrm{nF})\end{array}$ & $\begin{array}{c}V_{\text {piezo }} \\
\left(\mathrm{cm}^{3}\right)\end{array}$ & $\begin{array}{c}\alpha_{\mathrm{ME}} \\
(\mathrm{V} / \mathrm{cm} \mathrm{Oe})\end{array}$ \\
\hline 0 & Terfenol-D/PZT-5 & Unimorph & 2.43 & 0.045 & 0.5 \\
1 & Terfenol-D/PZT-5 & Unimorph & 2.04 & 0.216 & 0.56 \\
2 & Terfenol-D/PZT-5 & $L-T$ & 1.9 & 0.047 & 2 \\
3 & Terfenol-D/PZT-5 & $L-T$ & 3.81 & 0.094 & 2 \\
4 & Terfenol-D/PZT-5 & $L-T$ & 1.95 & 0.216 & 2.17 \\
5 & Terfenol-D/PZT-5 & $L-T$ & 3.7 & 0.4 & 2.1 \\
6 & Terfenol-D/PZT-5 & $L-T P-P$ & 7.3 & 0.79 & 2.2 \\
\hline \hline
\end{tabular}

${ }^{\mathrm{a}} L-T$, Longitudinal-transverse; $P-P$, Push-pull.

$$
\begin{aligned}
E_{\mathrm{no}} & =\left[\int_{f_{L}}^{f_{H}} i_{t}^{2}(\omega)\left|Z_{n}(j \omega)\right|^{2} d f\right]^{0.5} \\
& =\sqrt{C}\left[\int_{f_{L}}^{f_{H}} A^{2}(\omega)\left|Z_{n}(j \omega)\right|^{2} d f\right]^{0.5}=B \sqrt{C}
\end{aligned}
$$

From Eq. (4), we can see that $E_{\text {no }}$ is proportional to $\sqrt{C}$. This suggests to minimize the total voltage noise that we simply need to decrease the capacitance of the sensor. However, this is slightly misleading, as we also need to have a large ME signal-it is the SNR that matters for high signal sensitivity.

Now, assume that the signal gain at a specific frequency $\omega_{0}$ is $G\left(\omega_{0}\right)$. From the equivalent circuit in Fig. 1, we can acquire that the output me voltage is $V_{o}=j \omega_{0} \alpha_{\mathrm{ME}} H G\left(\omega_{0}\right) .{ }^{10}$ The me charge coefficient $\left(\alpha_{\mathrm{ME}}\right)$ can be obtained from ME voltage coefficient $\left(\alpha_{\mathrm{ME}}\right)$ as $\alpha_{\mathrm{me}}=\alpha_{\mathrm{ME}} / C t$, where $t$ is the thickness of the piezoelectric layer. So we can acquire the SNR at this specific frequency as

$$
\begin{aligned}
\mathrm{SNR} & =\frac{V_{o}}{E_{\mathrm{no}}}=\frac{j \omega_{0} H\left(\omega_{0}\right) G\left(\omega_{0}\right)}{B} \sqrt{C} t \alpha_{\mathrm{ME}} \\
& =\frac{j \omega_{0} H\left(\omega_{0}\right) G\left(\omega_{0}\right)}{B} \sqrt{\varepsilon V} \alpha_{\mathrm{ME}},
\end{aligned}
$$

where $V$ is the volume of the piezoelectric phase. Previous reports have shown that in one mode, the $\alpha_{\mathrm{ME}}$ will keep the same if we keep the volume fraction of the two phases of the ME laminates unchanged, ${ }^{8,19}$ and from Eq. (5), we can draw a conclusion. If, in one mode, we keep the volume fraction of the two phases of the ME laminate constant, while changing the total volume by a factor of $m$, the SNR will be increased by a factor of $\sqrt{m}$ : we designate this as the "SNR scale effect" or "sensitivity scale effect."

We then constructed a number of ME laminate sensors made from Terfenol-D/PZT that operated in different couple modes [unimorph, longitudinal-transverse $(L-T)$, and pushpull $(P-P)],{ }^{6,9,20}$ and that had different volumes of piezoelectric phase $\left(V_{\text {piezo }}\right)$. In Table I, we list each of the ME sensors that we constructed with the corresponding values of $V_{\text {piezo }}$, $C$, and $\alpha_{\mathrm{ME}}$. Measurements of the output noise and sensitivity limit were carried out by placing the ME laminates in a Helmholz coil, and applying a dc magnetic bias $\left(H_{\mathrm{dc}}\right)$ and a small low frequency ac magnetic field $\left(H_{\mathrm{ac}}\right)$ along the longitudinal direction of the laminates. To measure the output noise and sensitivity limit, a low noise charge amplifier detection circuit was constructed, which had a gain of $1 \mathrm{~V} / \mathrm{pC}$ over the frequency range of $0.16-10 \mathrm{~Hz}$. The noise level of the amplifier was $<4 \mathrm{fA}_{\mathrm{p}-\mathrm{p} \text {. }}$ referenced to the input or $<4 \mathrm{mV}$ referenced to the output.
We next measured the output noise level $\left(E_{\mathrm{no}}\right)$ from each of the sensors listed in Table I, using the said detection circuitry. In Fig. 2(a), we plot $E_{\text {no }}$ for various sensors as a function of their respective values of $\sqrt{C}$. In this figure, we can see that there is a near linear relationship between $E_{\text {no }}$ and $\sqrt{C}$, as predicted above by Eq. (4). Next, in Fig. 2(b), we show the SNR as a function of $\sqrt{V} \alpha_{\mathrm{ME}}$, where a linear relationship can again be seen, consistent with the predictions of Eq. (5). These results show (i) that the output noise level and the SNR are nearly independent on the operational mode, (ii) that practically $E_{\text {no }}$ is determined by the laminate capacitance, and (iii) that the SNR has a scale effect, where it can be increased simply by increasing the volume of the sensor.

As an example of this scale effect, the waveforms in real time of the output noise and lowest detectable magnetic field are shown in Figs. 3(a) and 3(b), respectively. Data are shown for both small (No. 2 in Table I) and large (No. 5)
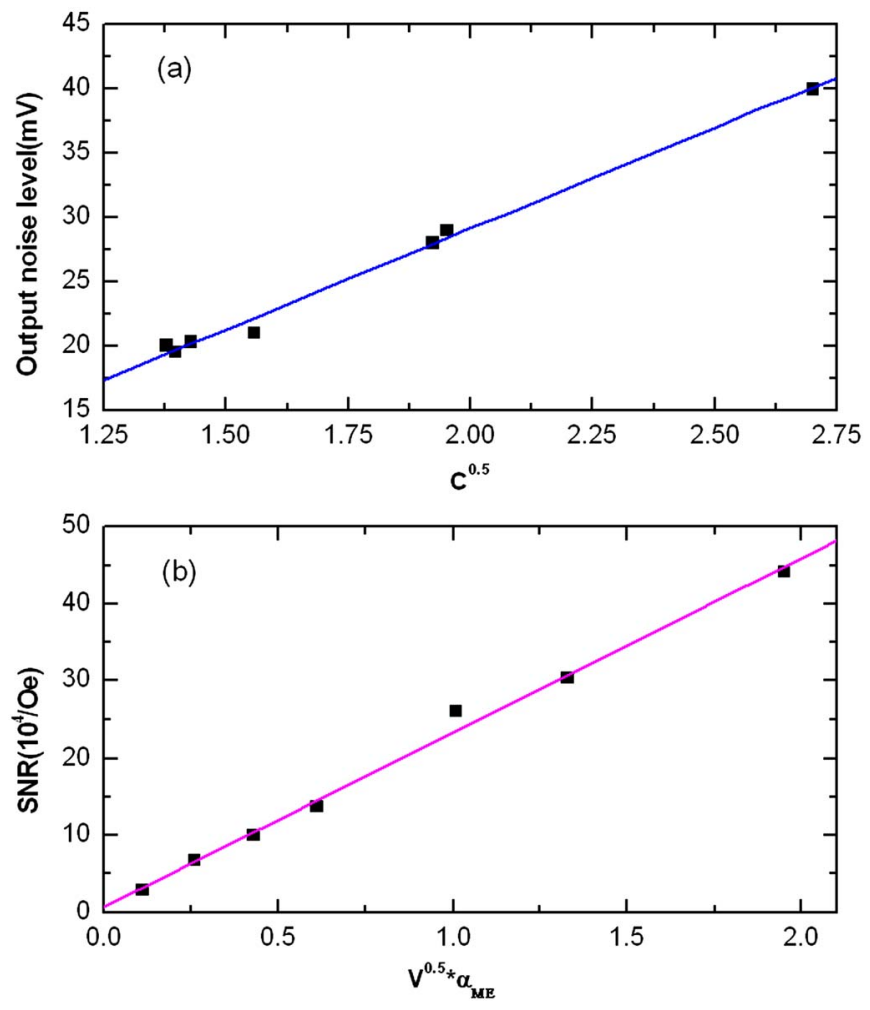

FIG. 2. (Color online) Dependence of (a) the output noise level $\left(E_{\mathrm{no}}\right)$ on sensor capacitance $(C)$ for the various laminate composites given in Table I, and (b) the signal-to-noise ratio (SNR) on $\sqrt{V} \alpha_{\mathrm{ME}}$, where $V$ is the volume of the piezoelectric phase and $\alpha_{\mathrm{ME}}$ is the magnetoelectric voltage coefficient. Each point corresponds to one of the ME laminate sensors enumerated in Table I. 
(a)

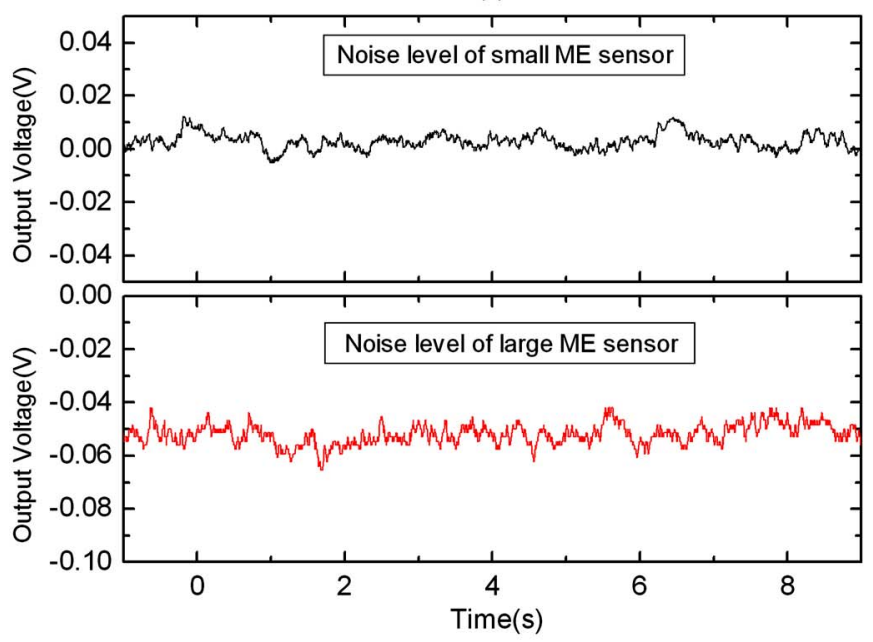

(b)

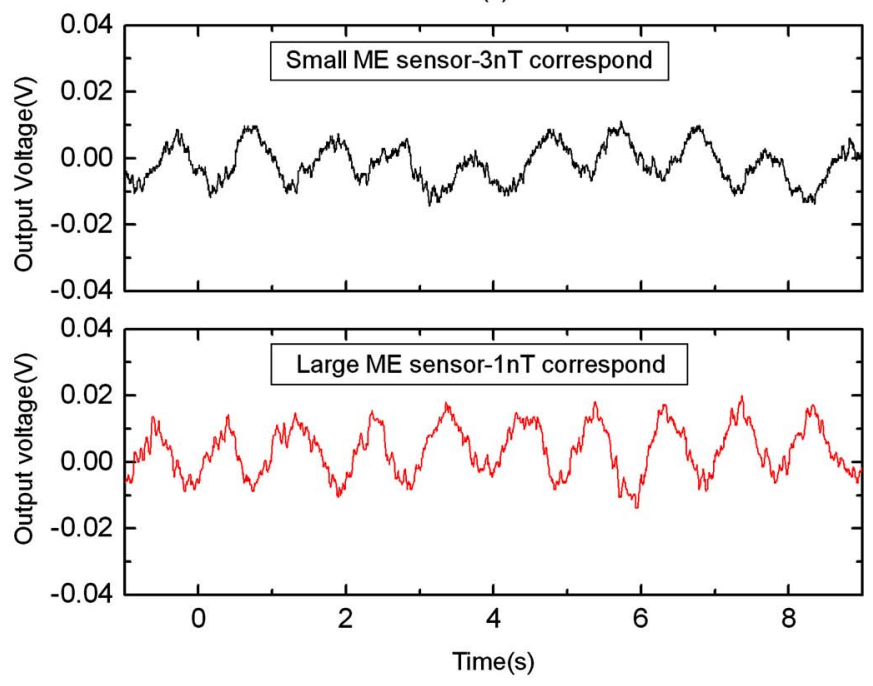

FIG. 3. (Color online) Waveforms in real time of (a) the output noise level $\left(E_{\mathrm{no}}\right)$ of a small $L-T$ mode ME laminate (sensor No. 2 in Table I) and of a large one (sensor No. 5), and (b) the lowest detectable magnetic field $(f=1 \mathrm{~Hz})$. The small ME sensor had a sensitivity limit of $\sim 3 \mathrm{nT}$, whereas the large ME sensor had $\sim 1 \mathrm{nT}$.

sensors in each figure part. The output noise level of the small ME sensor was $\sim 20 \mathrm{mV}$, while the large one was $\sim 30 \mathrm{mV}$. The lowest detective magnetic field of the small $\mathrm{ME}$ sensor is about $3 \mathrm{nT}$, while the large one can achieve $1 \mathrm{nT}$ magnetic field sensitivity. Finally, in Fig. 4, we plot the lowest detectable magnetic signal as a function of $(1 / \sqrt{V}) \alpha_{\mathrm{ME}}$ for various ME laminates. The linear trends in these results demonstrate that to detect smaller magnetic fields, one can either increase the sensor volume $V$ or the value of ME voltage coefficient $\alpha_{\mathrm{ME}}$.

In summary, the noise and the SNR of various ME sensors has been investigated. Theory predicts and experimental results confirm for various ME sensors (using the same de-

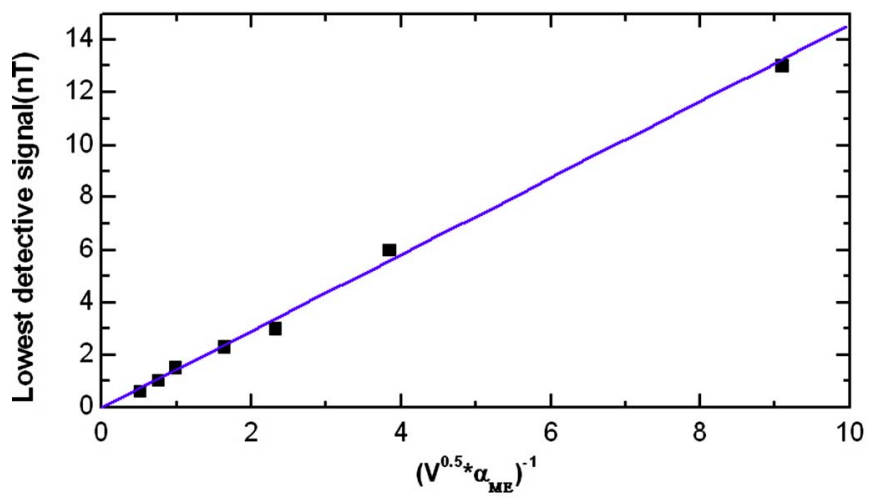

FIG. 4. (Color online) The lowest detectable magnetic field $(f=1 \mathrm{~Hz})$ on $(1 / \sqrt{V})_{\alpha_{\mathrm{ME}}}$

tection circuitry) that the noise is proportional to $\sqrt{C}$ and that the SNR is proportional to $\sqrt{V} \alpha_{\mathrm{ME}}$. The findings provide insights in how to enhanced sensitivity to small variations in magnetic fields that can be achieved in laminate composites of magnetostrictive and piezoelectric layers.

We are grateful to the Office of Naval Research for supporting this research effort.

${ }^{1}$ L. D. Landau and E. M. Lifshitz, Electrodynamics of Continuous Media (Pergamon, Oxford, 1960), Chap. 4, p. 119.

${ }^{2}$ M. Fiebig, J. Phys. D 38, 123 (2005).

${ }^{3}$ J. Wang, J. B. Neaton, H. Zheng, V. Nagarajan, S. B. Ogale, B. Liu, D. Viehland, V. Vaithyanathan, D. G. Schlom, U. V. Waghmare, N. A. Spaldin, K. M. Rabe, M. Wuttig, and R. Ramesh, Science 299, 1719 (2003).

${ }^{4}$ C. W. Nan, Phys. Rev. B 50, 6082 (1994).

${ }^{5}$ J. Ryu, C. Z. Carazo, K. Uchino, and H. E. Kim, Jpn. J. Appl. Phys., Part 1 40, 4948 (2001)

${ }^{6}$ S. X. Dong, J. F. Li, and D. Viehland, Appl. Phys. Lett. 83, 2265 (2003).

${ }^{7}$ G. Srinivasan, R. Hayes, and C. P. Devreugd, Appl. Phys. A: Mater. Sci. Process. 80, 891 (2005).

${ }^{8}$ M. I. Bichurin, D. A. Filippov, and V. M. Petrov, Phys. Rev. B 68, 054402 (2003).

${ }^{9}$ Z. Xing, S. X. dong, J. Zhai, L. Yan, J. F. Li, and D. Viehland, Appl. Phys. Lett. 89, 112911 (2006).

${ }^{10}$ Z. Xing, J. F. Li, and D. Viehland, Appl. Phys. Lett. 91, 142905, 2007.

${ }^{11}$ Z. Xing, J. Zhai, S. X. Dong, J. F. Li, D. Viehland, and W. G. Odendaal, Modeling and Detection of Quasi-Static Nano-Tesla Magnetic Field Variations Using Magneto-Electric Laminate Sensors (Dirac House, Bristol, England) (to be published).

${ }^{12}$ S. X. Dong, J. Zhai, Z. Xing, J. F. Li, and D. Viehland, Appl. Phys. Lett. 86, 102901 (2005).

${ }^{13}$ C. D. Motchenbacher and J. A. Connelly, Low Noise Electronic System Design (Wiley, New York, 1993), Chap. 1, p. 18.

${ }^{14}$ E. H. Putley, Semiconductors and Semimetals (Academic, New York, 1970), Vol. 5, Chap. 6, p. 267.

${ }^{15}$ S. G. Porter, Ferroelectrics 33, 193 (1981).

${ }^{16}$ A. Hossain and M. H. Rashid, IEEE Trans. Ind. Appl. 27, 824 (1991).

${ }^{17}$ S. E. Stokowski, Appl. Phys. Lett. 29, 393 (1976).

${ }^{18}$ Sergio Franco, Design with Operational Amplifiers and Analog Integrated Circuits (McGraw-Hill, New York, 2002), Chap. 7, p. 318.

${ }^{19}$ M. I. Bichurin' and V. M. Petrov, J. Appl. Phys. 92, 7681 (2002).

${ }^{20}$ S. X. Dong, J. Zhai, F. Bai, J. F. Li, and D. Viehland, Appl. Phys. Lett. 87, 062502 (2005). 